

\title{
Ideals of degree one contribute most of the height
}

\author{
Aaron Levin and David McKinnon
}

\begin{abstract}
Let $k$ be a number field, $f(x) \in k[x]$ a polynomial over $k$ with $f(0) \neq 0$, and $O_{k, S}^{*}$ the group of $S$-units of $k$, where $S$ is an appropriate finite set of places of $k$. In this note, we prove that outside of some natural exceptional set $T \subset \mathrm{O}_{k, S}^{*}$, the prime ideals of $\mathcal{O}_{k}$ dividing $f(u), u \in \mathbb{O}_{k, S}^{*} \backslash T$, mostly have degree one over $\mathbb{Q}$; that is, the corresponding residue fields have degree one over the prime field. We also formulate a conjectural analogue of this result for rational points on an elliptic curve over a number field, and deduce our conjecture from Vojta's conjecture. We prove this conjectural analogue in certain cases when the elliptic curve has complex multiplication.
\end{abstract}

\section{Introduction}

If $a$ is an algebraic integer in a number field $k$ and $f(x) \in \mathcal{O}_{k}[x]$ a polynomial, then the ideals dividing $f(a)$ are simply the ideals $I$ such that $f(a) \equiv 0(\bmod I)$. Heuristically, the larger the cardinality of the residue ring $O_{k} / I$, the smaller the probability that $f(a)$ and 0 are the same.

The purpose of this paper is to make this notion precise, to generalize it, and to prove it in the case described above. More specifically, in Theorem 2.1, using a result of Corvaja and Zannier, we prove a precise version of this notion for $\mathbb{G}_{m}$, and in Theorem 3.4, we state a conjectural analogue of Theorem 2.1 for elliptic curves over a number field, and show that it is a consequence of Vojta's conjecture [1987; 2011].

A theorem of the second author proves Vojta's conjecture in a relevant special case, and we deduce an unconditional version of Theorem 3.4 in that case. Specifically, if the elliptic curve $E / k$ has complex multiplication, and if the algebraic point $P$ is defined over the compositum of $k$ with $\operatorname{End}(E) \otimes \mathbb{Q}$, then we can deduce Theorem 3.4 without the hypothesis that Vojta's conjectures are true.

MSC2010: primary 11G50; secondary 11J25.

Keywords: heights, Diophantine approximation, polynomial values, elliptic curves, Vojta's conjecture. 


\section{Main theorem}

Let $f(x) \in k[x]$ be a polynomial over a number field $k$. The heuristic mentioned in the introduction suggests that a prime $\mathfrak{p}$ of $k$ is likelier to divide $f(a)$ for $a \in k$ if the residue field $O_{k} / \mathfrak{p}$ is small. Our main theorem will give one possible precise interpretation of this notion, where we view $\mathcal{O}_{k} / \mathfrak{p}$ as being small if $O_{k} / \mathfrak{p}$ has degree one over its prime field. There is, however, an obvious way that our heuristic can fail. Suppose, for example, that $f$ and $a$, and hence $f(a)$, are actually defined over a proper subfield $k^{\prime}$ of $k$. Then the size of $\mathcal{O}_{k^{\prime}} /\left(\mathfrak{p} \cap \mathcal{O}_{k^{\prime}}\right)$, and not $\mathcal{O}_{k} / \mathfrak{p}$, is clearly the relevant quantity. In the simplest case, when $k / \mathbb{Q}$ is Galois and $f$ is irreducible, our main theorem says, in essence, that for $S$-units $u$ of $k$ this is in fact the only way our heuristic can fail, that is, $f(u)$ is "mostly" supported on primes of $k$ of degree one over $\mathbb{Q}$ unless $f(u)$ is rational, in an appropriate sense, over a proper subfield of $k$.

The statement of the main theorem requires a fair amount of notation. We summarize this notation as follows:

\begin{tabular}{|c|c|}
\hline$k$ & Extension of $\mathbb{Q}$ of degree $d \neq 1$ \\
\hline$L$ & Galois closure of $k$ over $\mathbb{Q}$ \\
\hline $\operatorname{Gal}(L / \mathbb{Q})$ & The Galois group of $L$ over $\mathbb{Q}$ \\
\hline $\mathrm{O}_{k}$ & Ring of integers of $k$ \\
\hline$f(x)$ & Nonconstant polynomial in $\mathbb{O}_{k}[x]$ with $f(0) \neq 0$ \\
\hline$f_{1}, \ldots, f_{N}$ & The monic irreducible factors of $f$ over $L$ \\
\hline$S$ & $\begin{array}{l}\text { Finite set of places of } k \text { containing the archimedean places } \\
\text { such that if } v \in S \text { and } v \text { and } v^{\prime} \text { lie above the same rational } \\
\text { prime } p \in \mathbb{Z} \text { then } v^{\prime} \in S \text {. }\end{array}$ \\
\hline $\mathrm{O}_{k, S}$ & Ring of $S$-integers of $k$ \\
\hline $\mathrm{O}_{k, S}^{*}$ & Group of $S$-units of $k$ \\
\hline$\tau$ & The involution $\tau(u)=u^{-1}$ of $\mathrm{O}_{k, S}^{*}$. \\
\hline $\mathrm{O}_{k, S}^{* \phi}$ & $\begin{array}{l}\text { For a homomorphism } \phi \text {, the subgroup of } O_{k, S}^{*} \text { consisting of } \\
\text { elements } u \text { such that } \phi(u)=u \text {. }\end{array}$ \\
\hline$I(f(u))$ & The ideal generated by $f(u)$ in the ring $O_{k, S}$ \\
\hline$J(f(u))$ & $\begin{array}{l}\text { Smallest ideal dividing } I(f(u)) \text { such that for every prime } \\
\mathfrak{p} \text { dividing } J(f(u)), \mathbb{O}_{k, S} / \mathfrak{p} \text { has degree greater than one } \\
\text { over the prime field }\end{array}$ \\
\hline$N(I)$ & The norm of $I$ over $\mathbb{Q}$, for any ideal $I$ of $\mathscr{O}_{k}$ or $\mathscr{O}_{k, S}$ \\
\hline$H_{k}(x)$ & The relative multiplicative Weil height of $x \in k$ \\
\hline$H(x)$ & $\begin{array}{l}\text { The absolute multiplicative Weil height of } x \text {, } \\
\text { equal to } H_{k}(x)^{1 / d} \text { for } x \in k\end{array}$ \\
\hline$h(x)$ & The absolute logarithmic Weil height of $x$, equal to $\log H(x)$ \\
\hline
\end{tabular}


We can now state the main theorem:

Theorem 2.1. Let $\epsilon>0$. Let $f(x) \in \mathbb{O}_{k}[x]$ satisfy $f(0) \neq 0$. Then there exists a finite set of places $S^{\prime}$ of $L$ such that for every $u \in \mathbb{O}_{k, S}^{*}$ either

$$
N(J(f(u)))<H(u)^{\epsilon}
$$

or

$$
f_{i}(u) \mathscr{O}_{L, S^{\prime}}=\alpha \mathfrak{O}_{L, S^{\prime}}
$$

for some $i$ and some $\alpha$ that lies in a proper subfield of $L$ not containing $k$ (in particular, if $k / \mathbb{Q}$ is Galois, $\alpha$ lies in a proper subfield of $k$ ).

With the exception of finitely many elements, the set of elements in $\mathrm{O}_{k, S}^{*}$ not satisfying (a) is contained in a finite union of cosets in $\mathrm{O}_{k, S}^{*}$ of the form

$$
T=u_{1} \mathcal{O}_{k, S}^{* \sigma_{1}} \cup \cdots \cup u_{m^{\prime}} \mathrm{O}_{k, S}^{* \sigma_{m^{\prime}}} \cup u_{m^{\prime}+1} \mathrm{O}_{k, S}^{* \sigma_{m^{\prime}+1} \tau} \cup \cdots \cup u_{m} \mathrm{O}_{k, S}^{* \sigma_{m} \tau},
$$

where $u_{1}, \ldots, u_{m} \in \mathbb{O}_{k, S}^{*}$ and $\sigma_{1}, \ldots, \sigma_{m} \in \operatorname{Gal}(L / \mathbb{Q}) \backslash \mathrm{Gal}(L / k)$ (not necessarily distinct) are effectively computable.

An alternative formulation of Theorem 2.1 involving only heights is given in Corollary 2.6. We mention also that the group $0_{k, S}^{* \sigma_{i}}$ is the same as $0_{F, S_{F}}^{*}$, where $F$ is the fixed field of $\sigma_{i}$ and $S_{F}$ is the set of places of $F$ lying below places of $S$.

Note that $H(f(u)) \ll H(u)^{\operatorname{deg} f}$ and that

$$
H_{k}(f(u))=C_{u} N(I(f(u)))=C_{u} N(J(f(u))) N(I(f(u)) / J(f(u))),
$$

where $C_{u}$ is a real number (roughly equal to the archimedean part of the height of $f(u)^{-1}$ ) satisfying $C_{u} \ll H(u)^{\epsilon}$ (see Lemma 2.7). Thus, Theorem 2.1 implies that for $u \in \mathbb{O}_{k, S}^{*} \backslash T, f(u)$ is "mostly" supported on primes of $k$ of degree one over $\mathbb{Q}$.

Finally, let us mention some possible generalizations of Theorem 2.1. Firstly, we note that for any integer $n$ and $u \in 0_{k, S}^{*}$, we have $J\left(u^{n} f(u)\right)=J(f(u))$ and $u^{n} f(u) \mathcal{O}_{k, S}=f(u) \mathscr{O}_{k, S}$. Thus, Theorem 2.1 immediately extends to Laurent polynomials (that is, $f(x) \in k[x, 1 / x]$ ). However, if $f(x)$ has a zero or pole at $x=0$, then the interpretation that for $u \in \mathbb{O}_{k, S}^{*} \backslash T, f(u)$ is "mostly" supported on primes of $k$ of degree one over $\mathbb{Q}$ is no longer necessarily valid (the inequality $N(I(f(u))) \gg H_{k}(f(u))^{1-\epsilon}$ may not hold in the previous remark). More generally, Theorem 2.1 may be extended in a straightforward way to rational functions (appropriately using fractional ideals in place of integral ideals). In a different direction, it seems to be an interesting problem to formulate an appropriate generalization of Theorem 2.1 that is valid for $S$-integers (as opposed to just $S$-units), or to prove a multivariable analogue.

Before we begin the proof, we introduce some notation. For a number field $k$ we denote the set of inequivalent places of $k$ by $M_{k}$. We define the function $\log ^{-}$ for positive real numbers $x$ by $\log ^{-}(x)=\min \{0, \log (x)\}$. For a place $v \in M_{k}$, we 
normalize the corresponding absolute value $|\cdot|_{v}$ in such a way that the product formula holds and $H(x)=\prod_{v \in M_{k}} \max \left\{1,|x|_{v}\right\}$.

Proof of Theorem 2.1. Consider the set

$$
U=\left\{u \in \mathbb{O}_{k, S}^{*} \mid N(J(f(u))) \geq H(u)^{\epsilon}\right\} .
$$

Let $L$ be a Galois closure of $k$ over $\mathbb{Q}$. Let $\mathfrak{p}$ be a prime of $O_{k}$ of inertia degree greater than one over $\mathbb{Q}$, lying above a rational prime $p \in \mathbb{Z}$. Let $\mathfrak{q}$ be a prime of $\mathcal{O}_{L}$ lying above $\mathfrak{p}$. Then $\mathfrak{q}$ again has inertia degree greater than one over $\mathbb{Q}$. Let $D=D(\mathfrak{q} / p) \subset \operatorname{Gal}(L / \mathbb{Q})$ be the decomposition group of $\mathfrak{q}$ and let $L^{D}$ be the decomposition field. Then $k \not \subset L^{D}$ since $\mathfrak{p}$ has inertia degree greater than one. It follows that there exists $\sigma \in \operatorname{Gal}(L / \mathbb{Q})$ such that $\sigma(\mathfrak{q})=\mathfrak{q}, \sigma \notin \operatorname{Gal}(L / k)$.

Let $S_{L}$ be the set of places of $L$ lying above places of $S$. Let

$$
J^{\prime}(f(u))=J(f(u)) \mathcal{O}_{L, S_{L}} .
$$

Let $\mathfrak{q}$ be a prime of $\mathrm{O}_{L, S_{L}}$ dividing $J^{\prime}(f(u))$. From the above discussion and the definition of $J(f(u))$, there exists an element $\sigma \in \operatorname{Gal}(L / \mathbb{Q}) \backslash \mathrm{Gal}(L / k)$ such that $\sigma(\mathfrak{q})=\mathfrak{q}$. Let $\operatorname{Gal}(L / \mathbb{Q}) \backslash \operatorname{Gal}(L / k)=\left\{\sigma_{1}, \ldots, \sigma_{m}\right\}$. For $i=1, \ldots, m$, define the ideal $J_{i}^{\prime}(f(u))$ to be the smallest ideal of $O_{L, S_{L}}$ dividing $J^{\prime}(f(u))$ such that $\sigma_{i}\left(J_{i}^{\prime}(f(u))\right)=J_{i}^{\prime}(f(u))$. Then $J^{\prime}(f(u))$ divides $J_{1}^{\prime}(f(u)) \cdots J_{m}^{\prime}(f(u))$. Note also that $N\left(J^{\prime}(f(u))\right) \geq N(J(f(u)))$. Let

$$
U_{i}=\left\{u \in U \mid N\left(J_{i}^{\prime}(f(u))\right) \geq H(u)^{\epsilon / m}\right\} .
$$

Then clearly $U \subset \bigcup_{i=1}^{m} U_{i}$.

Let $r \in\{1, \ldots, m\}$, and let $f^{\sigma_{r}}$ denote the image of $f$ under the natural action of $\sigma_{r}$. By definition, $J_{r}^{\prime}(f(u))$ divides both $f(u) \mathcal{O}_{L, S_{L}}$ and $f^{\sigma_{r}}\left(\sigma_{r}(u)\right) \mathcal{O}_{L, S_{L}}$ for all $u$. For $u \in U_{r}$, we therefore obtain

$$
\begin{aligned}
{[L: \mathbb{Q}] \sum_{v \in M_{L}} \log ^{-} \max \left\{|f(u)|_{v},\left|f^{\sigma_{r}}\left(\sigma_{r}(u)\right)\right|_{v}\right\} } & \leq-\log N\left(J_{r}^{\prime}(f(u))\right) \\
& \leq-\log H(u)^{\epsilon / m} \leq-\frac{\epsilon}{m} h(u) .
\end{aligned}
$$

Theorem 2.1 will follow essentially from the following:

Lemma 2.2 [Corvaja and Zannier 2005, Proposition 4]. Let $f(x), g(x) \in L[x]$ be polynomials that do not vanish at $x=0$. Then, for every $\epsilon>0$, all but finitely many solutions $\left(u, u^{\prime}\right) \in\left(\mathrm{O}_{L, S_{L}}^{*}\right)^{2}$ to the inequality

$$
\sum_{v \in M_{L}} \log ^{-} \max \left\{|f(u)|_{v},\left|g\left(u^{\prime}\right)\right|_{v}\right\}<-\epsilon\left(\max \left\{h(u), h\left(u^{\prime}\right)\right\}\right)
$$

are contained in finitely many effectively computable translates of one-dimensional subgroups of $\mathbb{G}_{m}^{2}$. 
Since $h(u)=h\left(\sigma_{r}(u)\right)$ and $u, \sigma_{r}(u) \in \mathcal{O}_{L, S_{L}}^{*}$, taking $g=f^{\sigma_{r}}$ it follows immediately from Lemma 2.2 that all but finitely many elements of the set

$$
V_{r}=\left\{\left(u, \sigma_{r}(u)\right) \mid u \in U_{r}\right\}
$$

are contained in finitely many effectively computable translates of one-dimensional subgroups of $\mathbb{G}_{m}^{2}$. Let $X$ be a translate of a one-dimensional subgroup of $\mathbb{G}_{m}^{2}$ that contains infinitely many elements of $V_{r}$. Let $\left(v, \sigma_{r}(v)\right) \in X \cap V_{r}$. Taking $u=v^{\prime} / v \in \mathbb{O}_{k, S}^{*}$, where $\left(v^{\prime}, \sigma_{r}\left(v^{\prime}\right)\right) \in X \cap V_{r}$, we see that infinitely many elements of the form $\left(u, \sigma_{r}(u)\right), u \in \mathbb{O}_{k, S}^{*}$, will lie in the associated one-dimensional subgroup in $\mathbb{G}_{m}^{2}$. We now classify the possibilities for such a one-dimensional subgroup.

Suppose there exists $a, b \in \mathbb{Z}$, not both zero, such that

$$
u^{a} \sigma_{r}(u)^{b}=1,
$$

for infinitely many $u \in \mathcal{O}_{k, S}^{*}$. We claim that $a= \pm b$. Let $l$ be the order of $\sigma_{r}$. Then

$$
u^{b^{l}}=\sigma_{r}^{l}(u)^{b^{l}}=\sigma_{r}^{l-1}(u)^{-a b^{l-1}}=\cdots=u^{(-a)^{l}} .
$$

So $u^{b^{l}-(-a)^{l}}=1$ for infinitely many $u \in \mathbb{O}_{k, S}^{*}$. This implies that $b^{l}=(-a)^{l}$, or $a= \pm b$, as claimed.

Suppose first that $a=-b$. Then for any $u \in 0_{k, S}^{*}$ satisfying (2) we have $\sigma_{r}\left(u^{a}\right)=$ $u^{a}$. So $u^{a} \in \mathcal{O}_{k, S}^{* \sigma_{r}}=F \cap \mathcal{O}_{k, S}^{*}$, where $F$ is the fixed field of $\sigma_{r}$. It follows that $\mathcal{O}_{k, S}^{* \sigma_{r}}$ has finite index in $\left\{u \in \mathcal{O}_{k, S}^{*} \mid u^{a} \sigma_{r}(u)^{-a}=1\right\}$ and that $\left\{u \in \mathcal{O}_{k, S}^{*} \mid\left(u, \sigma_{r}(u)\right) \in X \cap V_{r}\right\}$ is contained in a finite number of cosets of $O_{k, S}^{*} \sigma_{r}$ in $O_{k, S}^{*}$.

Suppose now that $a=b$. Then for any $u \in 0_{k, S}^{*}$ satisfying (2) we have $\sigma_{r}\left(u^{a}\right)=$ $u^{-a}$. By definition, we have $u^{-a} \in \mathcal{O}_{k, S}^{* \sigma_{r} \tau}$. Then, as above, we find that

$$
\left\{u \in \mathcal{O}_{k, S}^{*} \mid\left(u, \sigma_{r}(u)\right) \in X \cap V_{r}\right\}
$$

is contained in a finite number of cosets of $\mathcal{O}_{k, S}^{* \sigma_{r} \tau}$ in $O_{k, S}^{*}$.

Since there are only finitely many such $X$ and finitely many $r$, we conclude that there exists a set $T$ as in the statement of the theorem such that $U \backslash T$ is finite.

We now prove that all of the elements in $T$ satisfy (1) for some choice of $S^{\prime}$, completing the proof of the theorem. Let $f_{1}, \ldots, f_{N} \in L[x]$ be the monic irreducible factors of $f(x)$ over $L$. First, consider cosets in $O_{k, S}^{*}$ of the form $u_{i} \mathbb{O}_{k, S}^{* \sigma_{r}}$. From a slight modification of the first part of the proof above, we need only consider cosets $u_{i} \mathbb{O}_{k, S}^{* \sigma_{r}}$ such that for some $j \in\{1, \ldots, N\}$ and $\epsilon>0$, there are infinitely many $u \in \mathcal{O}_{k, S}^{* \sigma_{r}}$ such that

$$
\sum_{v \in M_{L}} \log ^{-} \max \left\{\left|f_{j}\left(u_{i} u\right)\right|_{v},\left|f_{j}^{\sigma_{r}}\left(\sigma_{r}\left(u_{i} u\right)\right)\right|_{v}\right\} \leq-\epsilon h\left(u_{i} u\right) .
$$


Note that $\sigma_{r}\left(u_{i} u\right)=\sigma_{r}\left(u_{i}\right) u$, since $u \in \mathcal{O}_{k, S}^{* \sigma_{r}}$. If $f_{j}\left(u_{i} x\right)$ and $f_{j}^{\sigma_{r}}\left(\sigma_{r}\left(u_{i}\right) x\right)$ are relatively prime in $L[x]$, then the left-hand side of (3) is bounded from below, independent of $u \in \mathcal{O}_{k, S}^{* \sigma_{r}}$. Since there are only finitely many $u \in \mathcal{O}_{k, S}^{* \sigma_{r}}$ with $h\left(u_{i} u\right)$ bounded, this contradicts the inequality (3) for all but finitely many $u \in \mathcal{O}_{k, S}^{* \sigma_{r}}$. So $f_{j}\left(u_{i} x\right)$ and $f_{j}^{\sigma_{r}}\left(\sigma_{r}\left(u_{i}\right) x\right)$ have a nontrivial common factor. Since $f_{j}\left(u_{i} x\right)$ and $f_{j}^{\sigma_{r}}\left(\sigma_{r}\left(u_{i}\right) x\right)$ are both irreducible over $L$, they must then be equal up to multiplication by a constant factor. Thus,

$$
\frac{f_{j}\left(u_{i} x\right)}{u_{i}^{e}}=\frac{f_{j}^{\sigma_{r}}\left(\sigma_{r}\left(u_{i}\right) x\right)}{\sigma_{r}\left(u_{i}\right)^{e}},
$$

where $e=\operatorname{deg} f_{j}$. It follows that for all $u$ in $\mathcal{O}_{k, S}^{* \sigma_{r}}$,

$$
\frac{f_{j}\left(u_{i} u\right)}{u_{i}^{e}}=\sigma_{r}\left(\frac{f_{j}\left(u_{i} u\right)}{u_{i}^{e}}\right) .
$$

So $f_{j}\left(u_{i} u\right) / u_{i}^{e} \in k^{\prime}$, the fixed field of $\sigma_{r}$. Then, for all $u \in \mathcal{O}_{k, S}^{* \sigma_{r}}, f_{j}\left(u_{i} u\right) / u_{i}^{e}$ lies in a proper subfield of $L$ not containing $k$. So in this case (1) holds with $S^{\prime}=S_{L}$ (and $u$ replaced by $\left.u_{i} u\right)$.

Now consider a coset of the form $u_{i} \mathbb{O}_{k, S}^{* \sigma_{r} \tau}$. Again, we may assume that for some $j$ and some $\epsilon>0$, (3) is satisfied for infinitely many $u \in \mathcal{O}_{k, S}^{* \sigma_{r} \tau}$. By definition, for $u \in \mathcal{O}_{k, S}^{* \sigma_{r} \tau}$ we have $\sigma_{r}(u)=u^{-1}$. Let $e=\operatorname{deg} f_{j}$. Similar to before, if $f_{j}\left(u_{i} x\right)$ and $x^{e} f_{j}^{\sigma_{r}}\left(\sigma_{r}\left(u_{i}\right) / x\right)$ are relatively prime in $L[x]$, then it follows that

$$
\sum_{v \in M_{L}} \log ^{-} \max \left\{\left|f_{j}\left(u_{i} u\right)\right|_{v},\left|f_{j}^{\sigma_{r}}\left(\sigma_{r}\left(u_{i}\right) / u\right)\right|_{v}\right\}
$$

is bounded from below, independent of $u \in \mathcal{O}_{k, S}^{* \sigma_{r} \tau}$. This again gives a contradiction with (3) and so $f_{j}\left(u_{i} x\right)$ and $x^{e} f_{j}^{\sigma_{r}}\left(\sigma_{r}\left(u_{i}\right) / x\right)$ must have a nontrivial common factor over $L$. Since $f_{j}$ is irreducible over $L$, the two polynomials must be equal up to multiplication by a constant. Evaluating at any $x=u^{\prime} \in \mathcal{O}_{k, S}^{* \sigma_{r} \tau}$ with $f_{j}\left(u_{i} u^{\prime}\right) \neq 0$, we find that we must have that

$$
\frac{f_{j}\left(u_{i} x\right)}{f_{j}\left(u_{i} u^{\prime}\right)}=\frac{x^{e} f_{j}^{\sigma_{r}}\left(\sigma_{r}\left(u_{i}\right) / x\right)}{u^{\prime e} \sigma_{r}\left(f_{j}\left(u_{i} u^{\prime}\right)\right)} .
$$

Since $\left(O_{k, S}^{* \sigma_{r} \tau}\right)^{2}$ has finite index in $\mathcal{O}_{k, S}^{* \sigma_{r} \tau}$, we can find a finitely many elements $u_{1}^{\prime}, \ldots, u_{l^{\prime}}^{\prime} \in \mathcal{O}_{k, S}^{* \sigma_{r} \tau}$ with $f_{j}\left(u_{i} u_{l}^{\prime}\right) \neq 0, l=1, \ldots, l^{\prime}$, and such that for any $u \in O_{k, S}^{* \sigma_{r} \tau}$, there exists some $l \in\left\{1, \ldots, l^{\prime}\right\}$ with $u / u_{l}^{\prime} \in\left(\mathcal{O}_{k, S}^{* \sigma_{r} \tau}\right)^{2}$. Let $u \in \mathcal{O}_{k, S}^{* \sigma_{r} \tau}$ and $u_{l}^{\prime}$ chosen as above. Then we have the identity

$$
\sigma_{r}\left(\left(\frac{u_{l}^{\prime}}{u}\right)^{e / 2} \frac{f_{j}\left(u_{i} u\right)}{f_{j}\left(u_{i} u_{l}^{\prime}\right)}\right)=\left(\frac{u_{l}^{\prime}}{u}\right)^{e / 2} \frac{f_{j}\left(u_{i} u\right)}{f_{j}\left(u_{i} u_{l}^{\prime}\right)}
$$


and it follows that $\left(u_{l}^{\prime} / u\right)^{e / 2} f_{j}\left(u_{i} u\right) / f_{j}\left(u_{i} u_{l}^{\prime}\right) \in k^{\prime}$, the fixed field of $\sigma_{r}$. We can enlarge $S_{L}$ to a finite set of places $S^{\prime}$ of $L$ such that $f_{j}\left(u_{i} u_{l}^{\prime}\right)$ is an $S^{\prime}$-unit for all choices of $i, j$, and $l$. Then (1) holds for all $u \in u_{i} \mathcal{O}_{k, S}^{* \sigma_{r} \tau}$.

In the case of a cyclic subgroup of $k^{*}$ the theorem takes a particularly simple form.

Corollary 2.3. Let $a \in k^{*}$. Let $S$ be a finite set of places of $k$ such that $a$ is an $S$-unit. Assume that for all positive integers $m$,

(a) the element $a^{m}$ does not lie in a proper subfield of $k$, and

(b) $k$ is not a quadratic extension of a field $k^{\prime}$ with $N_{k^{\prime}}^{k}\left(a^{m}\right)=1$.

Let $\epsilon>0$ and let $f(x) \in \mathbb{O}_{k}[x]$ satisfy $f(0) \neq 0$. Then, for all but finitely many integers $n$,

$$
N\left(J\left(f\left(a^{n}\right)\right)\right)<H\left(a^{n}\right)^{\epsilon} .
$$

Proof. Suppose that for infinitely many $n, N\left(J\left(f\left(a^{n}\right)\right)\right) \geq H\left(a^{n}\right)^{\epsilon}$. Then by Theorem 2.1, there exists $\sigma \in \operatorname{Gal}(L / \mathbb{Q}) \backslash \operatorname{Gal}(k / \mathbb{Q})$ and $u \in \mathbb{O}_{k, S}^{*}$ such that for infinitely many $n, a^{n}$ lies in a coset of the form $u 0_{k, S}^{* \sigma}$ or $u 0_{k, S}^{* \sigma \tau}$. This implies that for some $m \neq 0, a^{m} \in \mathbb{O}_{k, S}^{* \sigma}$ or $a^{m} \in \mathcal{O}_{k, S}^{* \sigma \tau}$. In the first case, $a^{m}$ lies in the proper subfield $k \cap F$ of $k$, where $F$ is the fixed field of $\sigma$. Suppose that $a^{m} \in 0_{k, S}^{* \sigma \tau}$ and that $a^{m}$ does not lie in a proper subfield of $k$. Then $k=\mathbb{Q}\left(a^{m}\right)$. Since $\sigma\left(a^{m}\right)=a^{-m}$, $\sigma$ restricts to an automorphism of $k$ over $\mathbb{Q}$. Note that $\sigma^{2}\left(a^{m}\right)=a^{m}$, so $\sigma$ is an automorphism of $k$ of order 2. Let $k^{\prime}$ be the fixed field of $\sigma$. Then $\left[k: k^{\prime}\right]=2$, $\operatorname{Gal}\left(k / k^{\prime}\right)=\{\mathrm{id}, \sigma\}$, and $N_{k^{\prime}}^{k}\left(a^{m}\right)=a^{m} \sigma\left(a^{m}\right)=1$.

We give an example related to Fibonacci numbers to show the likely necessity of the less obvious condition (b) in Corollary 2.3.

Example 2.4. Let $k=\mathbb{Q}(\sqrt{5})$ and $a=\varphi=\frac{1+\sqrt{5}}{2} \in k^{*}$. Let $S$ consist of the archimedean places of $k$ and the prime lying above 5. Let $f(x)=x+1$. For $n$ odd, we have

$$
\frac{\varphi^{2 n}+1}{\varphi^{n} \sqrt{5}}=F_{n},
$$

where $F_{n}$ is the $n$-th Fibonacci number. So

$$
f\left(\varphi^{2 n}\right) \mathcal{O}_{k, S}=F_{n} \widehat{O}_{k, S} .
$$

A well-known naïve heuristic argument suggests that there should be infinitely many Fibonacci numbers that are prime and congruent to $\pm 2(\bmod 5)$ (so that these primes are inert in $k$ ). In this case, there would be an $\epsilon>0$ and infinitely many values of $n$ such that $N\left(J\left(f\left(\varphi^{n}\right)\right)\right)=N\left(f\left(\varphi^{n}\right)\right)>H\left(\varphi^{n}\right)^{\epsilon}$. This doesn't contradict Corollary 2.3 as $N_{\mathbb{Q}}^{k}\left(\varphi^{2}\right)=1$.

We now give a slight reformulation of our results. 
Definition 2.5. Let $D$ be an effective divisor on $\mathbb{P}^{1}$ defined over $k$ and supported on $\mathbb{P}^{1} \backslash\{0, \infty\}=\mathbb{G}_{m}$. Let $a \in k^{*}, a \notin \operatorname{Supp} D$, where Supp $D$ is the support of $D$. Let $h_{D}$ be the absolute logarithmic height associated to $D$ and let $h_{D}=\sum_{v \in M_{k}} h_{D, v}$ be a decomposition of $h_{D}$ into local heights (Weil functions). For a place $v \in M_{k}$ associated to a prime $\mathfrak{p}$ lying above a prime $p \in \mathbb{Z}$, let $f_{v}=f_{\mathfrak{p}}=\left[\mathcal{O}_{k} / \mathfrak{p}: \mathbb{Z} / p \mathbb{Z}\right]$. Set $f_{v}=1$ if $v \mid \infty$. We define the degree-one height of $a$ with respect to $k$ and $D$ by

Similarly, we define

$$
h_{D, \operatorname{deg}_{1}(k)}(a)=\sum_{\substack{v \in M_{k} \\ f_{v}=1}} h_{D, v}(a) .
$$

Note that

$$
h_{D, \operatorname{deg}_{>1}(k)}(a)=\sum_{\substack{v \in M_{k} \\ f_{v}>1}} h_{D, v}(a) .
$$

$$
h_{D}(a)=h_{D, \operatorname{deg}_{1}(k)}(a)+h_{D, \operatorname{deg}_{>1}(k)}(a)
$$

and by standard properties of heights, $h_{D, \operatorname{deg}_{1}(k)}$ and $h_{D, \operatorname{deg}_{>1}(k)}$ depend on the choice of $h_{D}$ and the local height functions only up to $O(1)$.

Corollary 2.6. Let $D$ be an effective divisor on $\mathbb{P}^{1}$ defined over $k$ and supported on $\mathbb{P}^{1} \backslash\{0, \infty\}$. Let $f(x) \in \mathbb{O}_{k}[x]$ be a polynomial defining $D$ with monic irreducible factors $f_{1}, \ldots, f_{n}$ over $L$. Let $\epsilon>0$. Then there exists a finite set of places $S^{\prime}$ of $L$ such that for every $u \in \mathbb{O}_{k, S}^{*}$ either

$$
h_{D, \operatorname{deg}_{>1}(k)}(u)<\epsilon h_{D}(u)
$$

or

$$
f_{i}(u) \mathfrak{O}_{L, S^{\prime}}=\alpha \mathfrak{O}_{L, S^{\prime}}
$$

for some $i$ and some $\alpha$ that lies in a proper subfield of $L$ not containing $k$.

All but finitely many elements not satisfying (a) are again contained in a set $T$ as in Theorem 2.1. There is also a similar reformulation of Corollary 2.3 in terms of $h_{D, \operatorname{deg}_{>1}(k)}(u)$.

Lemma 2.7. Let $D$ be as in Corollary 2.6. For any finite set of places $S^{\prime} \subset M_{k}$ and any $\epsilon>0$,

$$
\sum_{v \in S^{\prime}} h_{D, v}(u)<\epsilon h(u)+O(1)
$$

for all $u \in \mathbb{O}_{k, S}^{*}$

Proof. It suffices to show this for $D$ a point (not equal to 0 or $\infty$ ) and $S^{\prime} \supset S$. Let $E=0+\infty$. Since $u$ is an $S^{\prime}$-unit, we have

$$
\sum_{v \in S^{\prime}} h_{E, v}(u)=2 h(u)+O(1)
$$


By Roth's theorem,

$$
\sum_{v \in S^{\prime}} h_{D+E, v}(u)=\sum_{v \in S^{\prime}} h_{D, v}(u)+2 h(u)+O(1)<(2+\epsilon) h(u)+O(1),
$$

which gives (4).

In particular, it follows from Lemma 2.7 that Corollary 2.6 remains true if we add finitely many local heights to $h_{D, \text { deg }_{>1}(k)}$ (e.g., all the archimedean ones).

Proof of Corollary 2.6. We may take as local height functions associated to $D$ the functions

$$
h_{D, v}(a)=-\log ^{-}|f(a)|_{v}, \quad v \in M_{k} .
$$

Using Theorem 2.1 and Lemma 2.7, we can write, for all $u \in \mathbb{O}_{k, S}^{*}$,

$$
\begin{aligned}
h_{D, \operatorname{deg}_{>1}(k)}(u) & =\sum_{\substack{v \in M_{k} \\
f_{v}>1}} h_{D, v}(u)=-\sum_{\substack{v \in M_{k} \backslash S \\
f_{v}>1}} \log ^{-}|f(u)|_{v}+\sum_{\substack{v \in S \\
f_{v}>1}} h_{D, v}(u) \\
& =\frac{1}{[k: \mathbb{Q}]} \log N(J(f(u)))+\sum_{\substack{v \in S \\
f_{v}>1}} h_{D, v}(u) \\
& <\epsilon h(u)+O(1),
\end{aligned}
$$

\section{Elliptic curves}

Theorem 2.1 has a conjectural analogue for elliptic curves, following from a conjectural analogue of Lemma 2.2.

Conjecture 3.1 (Vojta). Let $E$ be an elliptic curve defined over a number field $k$. Let $h$ be an ample height function on $E$. Let $B \subset E(\bar{k}) \times E(\bar{k})$ be a finite set of points with $B$ defined over $k$. Let $\pi: X \rightarrow E \times E$ be the morphism obtained by blowing up the points in $B$ and let $Y$ be the exceptional divisor of $\pi$. Let $h_{Y}$ be a logarithmic height function with respect to $Y$. Let $\epsilon>0$. There exists a proper Zariski closed subset $Z(\epsilon)$ of $X$ such that for every $(P, Q) \in(E \times E)(k)-\pi(Z(\epsilon))$, we have

$$
h_{Y}\left(\pi^{-1}(P, Q)\right) \leq \epsilon(h(P)+h(Q))+O(1) .
$$

(Conjecture 3.1 is a special case of a much more general set of conjectures made by Vojta [1987; 2011].)

This enables us to deduce an analogue of Theorem 2.1 for elliptic curves. As in the previous section, it will be convenient to list the notation used: 


\begin{tabular}{|c|c|}
\hline$k$ & Fixed number field \\
\hline$\ell / k$ & Fixed nontrivial extension of $k$ \\
\hline$L$ & Galois closure of $\ell$ over $k$ \\
\hline $\operatorname{Gal}(L / k)$ & Galois group of $L$ over $k$ \\
\hline $\mathrm{O}_{k}$ & Ring of integers of $k$ \\
\hline$S$ & $\begin{array}{l}\text { Fixed finite set of places of } L \text {, consisting of the archimedean } \\
\text { places of } L \text { and the places of } L \text { ramified over } k\end{array}$ \\
\hline $\mathrm{O}_{L, S}$ & The ring of $S$-integers of $L$ \\
\hline E & $\begin{array}{l}\text { Fixed elliptic curve given by a Weierstrass equation } \\
y^{2}=x^{3}+a x+b, a, b \in \mathbb{O}_{k}\end{array}$ \\
\hline$E(\ell)^{\nu \sigma}$ & $\begin{array}{l}\text { For } v \in \operatorname{Aut}(E) \text { and } \sigma \in \operatorname{Gal}(L / k) \text {, the subgroup of } \\
\text { points } x \in E(\ell) \text { satisfying } v \sigma(x)=x\end{array}$ \\
\hline$D$ & Fixed effective and nontrivial $\ell$-rational divisor on $E$ \\
\hline$D_{1}, \ldots, D_{N}$ & The irreducible components of $D$ over $L$ \\
\hline$I_{D}(P)$ & Ideal associated to $D$ and $P$ (see Definition 3.2) \\
\hline$J_{D}(P)$ & $\begin{array}{l}\text { The smallest divisor ideal of } I_{D}(P) \text { supported on primes } \\
\mathfrak{p} \text { of } \mathscr{O}_{\ell} \text { with }\left[\mathscr{O}_{\ell} / \mathfrak{p}: \mathbb{O}_{k} /\left(\mathbb{O}_{k} \cap \mathfrak{p}\right)\right]>1\end{array}$ \\
\hline$N(I)$ & Absolute norm of an ideal $I$ of $O_{\ell}$ \\
\hline$H_{D}(P)$ & Multiplicative height function on $E$ corresponding to $D$ \\
\hline$h_{D}(P)$ & Logarithm of $H(P): h_{D}(P)=\log H_{D}(P)$. \\
\hline
\end{tabular}

Definition 3.2. Let $E: y^{2}=x^{3}+a x+b, a, b \in \mathbb{O}_{k}$, be an elliptic curve. Let $L$ be a number field containing $k$, and let $P, Q$ be distinct elements of $E(L)$. Let $P-Q=\left(x_{0}, y_{0}\right) \in E(L)$. Define

$$
I_{Q}(P)=\prod_{\mathfrak{p} \subset \mathscr{O}_{L}} \mathfrak{p}^{\max \left\{-\frac{1}{2} \operatorname{ord}_{\mathfrak{p}} x_{0}, 0\right\}},
$$

where $\mathfrak{p}$ runs over all (finite) primes of $\mathrm{O}_{L}$ (this is well-defined, independent of $L$, if we identify ideals $\mathfrak{a} \subset \mathfrak{O}_{L}$ and $\mathfrak{a} \mathfrak{O}_{L^{\prime}}$, when $\left.L \subset L^{\prime}\right)$. If $D=\sum_{i=1}^{n} Q_{i}, Q_{i} \in E(\bar{k})$, is a nontrivial effective divisor on $E$, then for $P \notin \operatorname{Supp}(D)$, we define

$$
I_{D}(P)=\prod_{i=1}^{n} I_{Q_{i}}(P) .
$$

Definition 3.3. Let $P \in E(\ell), P \notin \operatorname{Supp}(D)$. We define the height of $P$ with respect to degree-one primes of $\ell / k$ by

$$
h_{D, \operatorname{deg}_{1}(\ell / k)}(P)=\sum_{v \in M_{k}} \sum_{\substack{w \in M_{\ell} \\ w \mid v \\ f_{w / v}=1}} h_{D, w}(P),
$$


where $h_{D, w}$ denotes a local Weil height with respect to $D$ and $w$ and $f_{w / v}$ is the inertia degree of $w$ over $v$. Similarly, define

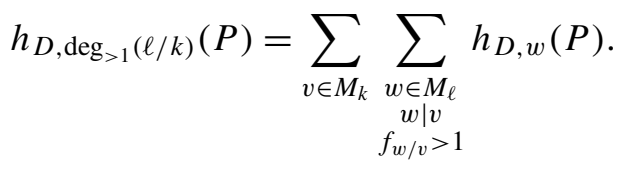

Note that, as in the previous section, we have

$$
h_{D, \operatorname{deg}_{1}(\ell / k)}(P)+h_{D, \operatorname{deg}_{>1}(\ell / k)}(P)=h_{D}(P)+O(1) .
$$

For $P \in E(\ell)$ and $D$ a divisor on $E$ defined over $\ell$, the norm $N\left(I_{D}(P)\right)$ is essentially just the nonarchimedean part of the (relative) height $H_{D, \ell}(P)=H_{D}(P)^{[\ell: \mathbb{Q}]}$ and $\log N\left(J_{D}(P)\right)=[\ell: \mathbb{Q}] h_{D, \operatorname{deg}_{>1}(\ell / k)}(P)$ (up to $O(1)$ ). We will assume the local heights are chosen so that this last statement is an equality.

We can now state the following theorem, which in the simplest case where $\ell / k$ is Galois, says, roughly, that the height of $P$ with respect to $D$ is "mostly" supported on the degree one primes of $\ell / k$, unless the ideal $I_{D}(P)$ is coming from a proper subfield of $\ell$. Note that the fields $\ell$ and $k$ here play the roles of $k$ and $\mathbb{Q}$, respectively, from the analogous Theorem 2.1.

Theorem 3.4. Let $\epsilon>0$. Assume that Conjecture 3.1 holds. Then, for every $P \in E(\ell)$, either

$$
\frac{1}{[\ell: \mathbb{Q}]} \log N\left(J_{D}(P)\right)=h_{D, \operatorname{deg}_{>1}(\ell / k)}(P)<\epsilon h_{D}(P),
$$

or

$$
I_{D_{i}}(P) \mathscr{O}_{L, S}=\mathfrak{a} \mathscr{O}_{L, S}
$$

for some $i$ and some ideal $\mathfrak{a} \subset \mathbb{O}_{k^{\prime}}$, where $k^{\prime}$ is a proper subfield of $L$ not containing $\ell$ (in particular, if $\ell / k$ is Galois, $\mathfrak{a}$ is contained in a proper subfield of $\ell$ ).

The set of points in $E(\ell)$ not satisfying (a) is contained in a finite union of cosets in $E(\ell)$ of the form

$$
T=\bigcup_{i=1}^{m} P_{i}+E(\ell)^{v_{i} \sigma_{i}},
$$

where $P_{i} \in E(\ell), \sigma_{i} \in \operatorname{Gal}(L / k) \backslash \operatorname{Gal}(L / \ell)$, and $v_{i} \in \operatorname{Aut}(E)$ for $i=1, \ldots, m$.

Proof. Let $D_{\text {red }}$ be the reduced divisor associated to $D$. Then, for some positive integer $c, D<c D_{\text {red }}$ and we have $h_{D}<c h_{D_{\text {red }}}+O(1)$ and $h_{D, \operatorname{deg}_{>1}(\ell / k)}<$ $c h_{D_{\text {red }}, \operatorname{deg}_{>1}(\ell / k)}+O(1)$. So without loss of generality we may assume that $D$ is a reduced divisor. Let

$$
U=\left\{P \in E(\ell) \mid h_{D, \operatorname{deg}_{>1}(\ell / k)}(P) \geq \epsilon h_{D}(P)\right\} .
$$


Let $L$ be a Galois closure of $\ell / k$. Let $w^{\prime} \in M_{L}$ lie above $w \in M_{\ell}$ and $v \in M_{k}$. As in the proof of Theorem 2.1, if $f_{w / v}>1$, then there exists $\sigma \in \operatorname{Gal}(L / k) \backslash \operatorname{Gal}(L / \ell)$ such that $\sigma\left(w^{\prime}\right)=w^{\prime}$. Let $\operatorname{Gal}(L / k) \backslash \operatorname{Gal}(L / \ell)=\left\{\sigma_{1}, \ldots, \sigma_{m}\right\}$. For $i=1, \ldots, m$, let

$$
h_{D, \operatorname{deg}_{>1}(L / k)}^{(i)}(P)=\sum_{v \in M_{k}} \sum_{\substack{w \in M_{L} \\ w \mid v, f_{w / v}>1 \\ \sigma_{i}(w)=w}} h_{D, w}(P) .
$$

Then

$$
h_{D, \operatorname{deg}_{>1}(\ell / k)}(P) \leq \sum_{i=1}^{m} h_{D, \operatorname{deg}_{>1}(L / k)}^{(i)}(P) .
$$

Let

$$
U_{i}=\left\{P \in U \mid h_{D, \operatorname{deg}_{>1}(L / k)}^{(i)}(P) \geq \frac{\epsilon}{m} h_{D}(P)\right\} .
$$

Then $U \subset \bigcup_{i=1}^{m} U_{i}$. Let $r \in\{1, \ldots, m\}$. If $w \in M_{L}$ and $\sigma_{r}(w)=w$, then $h_{D, w}(P)=$ $h_{\sigma_{r}(D), w}\left(\sigma_{r}(P)\right)$ and so

$$
\min \left\{h_{D, w}(P), h_{\sigma_{r}(D), w}\left(\sigma_{r}(P)\right)\right\}=h_{D, w}(P) .
$$

Let $\pi: X \rightarrow E \times E$ be the morphism obtained by blowing up the points in

$$
D \times \sigma_{r}(D) \subset E \times E
$$

and let $Y$ be the exceptional divisor of $\pi$. By well-known properties of heights, for $(P, Q) \notin D \times \sigma_{r}(D)$ and $w \in M_{L}$, we can choose

$$
h_{Y, w}\left(\pi^{-1}(P, Q)\right)=\min \left\{h_{D, w}(P), h_{\sigma_{r}(D), w}(Q)\right\} .
$$

Let $V_{r}=\left\{\left(P, \sigma_{r}(P)\right) \mid P \in U_{r}\right\}$. It follows that for $\left(P, \sigma_{r}(P)\right) \in V_{r}$, we have

$$
\begin{aligned}
h_{Y}\left(\pi^{-1}\left(P, \sigma_{r}(P)\right)\right) & \geq h_{D, \operatorname{deg}_{>1}(L / k)}^{(r)}(P) \geq \frac{\epsilon}{m} h_{D}(P) \\
& >\frac{\epsilon}{2 m}\left(h(P)+h\left(\sigma_{r}(P)\right)+O(1) .\right.
\end{aligned}
$$

Then by Conjecture 3.1 $V_{r}$ is contained in a proper Zariski closed subset of $E \times E$. If $V_{r}$ is a finite set, then $U_{r}$ is contained in a set $T$ as in the theorem. Otherwise, let $C$ be a positive-dimensional component of the Zariski closure of $V_{r}$. Then $C$ is a curve with infinitely many rational points on it. By Faltings' theorem, $C$ is a translate of a one-dimensional abelian subvariety $E^{\prime}$ of $E \times E$.

Any irreducible one-dimensional abelian subvariety of $E \times E$ must be an elliptic curve isogenous to $E$, via projection onto $E$. Since $E^{\prime}$ is clearly not a fiber of either of the two projection maps, there are two isogenies $\phi, \psi: E^{\prime} \rightarrow E$ induced by the two projection maps, with dual isogenies $\hat{\phi}$ and $\hat{\psi}$ from $E$ to $E^{\prime}$. If $R=(P, Q) \in$ $E^{\prime} \subset E \times E$, then $\hat{\phi} \phi(R)=\hat{\phi}(P)=(\operatorname{deg} \hat{\phi}) R$ and similarly $\hat{\psi}(Q)=(\operatorname{deg} \hat{\psi}) R$. 
Thus, $E^{\prime}$ is contained in the set $\{(P, Q) \in E \times E \mid(\operatorname{deg} \hat{\psi}) \hat{\phi}(P)=(\operatorname{deg} \hat{\phi}) \hat{\psi}(Q)\}$. Composing with an isogeny to $E$ we find that there are nonzero endomorphisms $f$ and $g$ of $E$ such that $E^{\prime} \subset\{(P, Q) \in E \times E \mid f(P)=g(Q)\}$. Note that if $\left(P_{0}, \sigma_{r}\left(P_{0}\right)\right),\left(P, \sigma_{r}(P)\right) \in V_{r} \cap C$ then $\left(P-P_{0}, \sigma_{r}\left(P-P_{0}\right)\right) \in E^{\prime}$. It follows that there are points of the form $\left(P, \sigma_{r}(P)\right) \in E^{\prime}$ with $P \in E(\ell)$ such that $f(P)=$ $g\left(\sigma_{r}(P)\right)$.

Let $K=\operatorname{End}_{L}(E) \otimes \mathbb{Q}$, where $\operatorname{End}_{L}(E)$ is the endomorphism ring of $E$ over $L$. Then $\sigma_{r}$ is an element of a finite group acting on the finite-dimensional $K$ vector space $V=E(L) \otimes_{\operatorname{End}_{L}(E)} K$. Thus, the eigenvalues of the action of $\sigma_{r}$ must be roots of unity. But from the above, $f / g$ is an eigenvalue of $\sigma_{r}$. So we deduce that $f / g \in K$ is a root of unity. Since $K$ is contained in a quadratic extension of $\mathbb{Q}$, this means that $f / g \in\left\{ \pm 1, \pm i, \pm \gamma, \pm \gamma^{2}\right\}$, where $\gamma$ denotes a primitive sixth root of unity. Write $g=v f$. Composing both sides with the dual endomorphism to $f$, we may assume that $f=m$, where $m$ is a positive integer. Then, for $\left(P, \sigma_{r}(P)\right),\left(P_{0}, \sigma_{r}\left(P_{0}\right)\right) \in V_{r} \cap C$, we have $m\left(P-P_{0}\right)=v \sigma_{r}\left(m\left(P-P_{0}\right)\right)$. This implies that $U_{r}$ is contained in finitely many cosets of the form $P_{i}+E(\ell)^{v_{i} \sigma_{r}}$ in $E(\ell)$, where $P_{i} \in E(\ell)$ and $v_{i} \in \operatorname{Aut}(E)$. So the set of points in $E(\ell)$ not satisfying (a) is contained in a set $T$ as in the theorem.

We now show that the set of points in the set $T$ not satisfying condition (a) satisfies condition (b). Let $D_{1}, \ldots, D_{N}$ be the irreducible components of $D$ over $L$. Consider a coset in $E(\ell)$ of the form $P_{r}+E(\ell)^{v_{r} \sigma_{r}}$, where $P_{r} \in E(\ell), v_{r} \in \operatorname{Aut}(E)$, and $\sigma_{r} \in \operatorname{Gal}(L / k) \backslash \operatorname{Gal}(L / \ell)$. From the first part of the proof, we need only consider cosets such that for some $i$, some $\epsilon>0$, and infinitely many elements $P \in E(\ell)^{v_{r} \sigma_{r}}$, we have

$$
\sum_{w \in M_{L}} \min \left\{h_{D_{i}, w}\left(P+P_{r}\right), h_{\sigma_{r}\left(D_{i}\right), w}\left(\sigma_{r}\left(P+P_{r}\right)\right)\right\}>\epsilon h(P) .
$$

Let $\phi: E \rightarrow E$ be the morphism $\phi(P)=v_{r}^{-1} P+\sigma_{r}\left(P_{r}\right)$. Since $\sigma_{r}\left(P+P_{r}\right)=$ $v_{r}^{-1} P+\sigma_{r}\left(P_{r}\right)$ for $P \in E(\ell)^{v_{r} \sigma_{r}}$, we have (up to $O(1)$ )

$$
h_{\sigma_{r}\left(D_{i}\right), w}\left(\sigma_{r}\left(P+P_{r}\right)\right)=h_{\sigma_{r}\left(D_{i}\right), w}(\phi(P))=h_{\phi^{*} \sigma_{r}\left(D_{i}\right), w}(P) .
$$

Let $\tau$ be translation by $P_{r}$. So $h_{D_{i}, w}\left(P+P_{r}\right)=h_{\tau^{*} D_{i}, w}(P)+O(1)$. So for infinitely many $P \in E(\ell)$,

$$
\sum_{w \in M_{L}} \min \left\{h_{\tau^{*} D_{i}, w}(P), h_{\phi^{*} \sigma_{r}\left(D_{i}\right), w}(P)\right\}>\epsilon h(P) .
$$

If $\tau^{*} D_{i}$ and $\phi^{*} \sigma_{r}\left(D_{i}\right)$ have empty intersection, then as is well known,

$$
\sum_{w \in M_{L}} \min \left\{h_{\tau^{*} D_{i}, w}(P), h_{\phi^{*} \sigma_{r}\left(D_{i}\right), w}(P)\right\}
$$


is bounded independent of $P$, contradicting (5). So $\tau^{*} D_{i} \cap \phi^{*} \sigma_{r}\left(D_{i}\right) \neq \varnothing$. Since $D_{i}$ is irreducible over $L$, this implies that $\tau^{*} D_{i}=\phi^{*} \sigma_{r}\left(D_{i}\right)$.

It follows from the definition that for any translation $\tau_{0}$ and any automorphism $v \in \operatorname{Aut}(E), I_{D}\left(\tau_{0}(P)\right)=I_{\tau_{0}^{*} D}(P)$ and $I_{D}(v P)=I_{v^{*} D}(P)$. This implies that for all $P \in E(\ell)^{v_{r} \sigma_{r}}$,

$$
\begin{aligned}
\sigma_{r}\left(I_{D_{i}}\left(P+P_{r}\right)\right) & =I_{\sigma_{r}\left(D_{i}\right)}\left(\sigma_{r}(P)+\sigma_{r}\left(P_{r}\right)\right)=I_{\sigma_{r}\left(D_{i}\right)}(\phi(P))=I_{\phi^{*} \sigma_{r}\left(D_{i}\right)}(P) \\
& =I_{\tau^{*} D_{i}}(P) \\
& =I_{D_{i}}\left(P+P_{r}\right) .
\end{aligned}
$$

So $\sigma_{r}$ fixes the ideal $I_{D_{i}}\left(P_{r}+P\right), P_{r}+P \in P_{r}+E(\ell)^{v_{r} \sigma_{r}}$, which implies that $I_{D_{i}}\left(P+P_{r}\right) \mathfrak{O}_{L, S}=\mathfrak{a} \mathfrak{O}_{L, S}$ for some ideal $\mathfrak{a}$ of $\mathcal{O}_{k^{\prime}}$, where $k^{\prime}$ is the fixed field of $\sigma_{r}$.

If we restrict to cyclic subgroups of $E(\ell)$, we obtain the following simpler version of Theorem 3.4.

Corollary 3.5. Let $P \in E(\ell)$ and $\epsilon>0$. If Conjecture 3.1 holds, then either

$$
h_{D, \operatorname{deg}_{>1}(\ell / k)}(n P)<\epsilon h_{D}(n P)
$$

for all but finitely many integers $n$, or there exists a proper subfield $k^{\prime} \varsubsetneqq \ell$ of $\ell, a$ positive integer $m$, an elliptic curve $E^{\prime} / k^{\prime}$, and an isomorphism $\phi: E \rightarrow E^{\prime}$ over $\ell$ such that $\phi(m P)$ is a $k^{\prime}$-rational point on $E^{\prime}$.

Proof. Suppose that for infinitely many $n, h_{D, \operatorname{deg}_{>1}(\ell / k)}(n P)<\epsilon h_{D}(n P)$. It follows from Theorem 3.4 that for some $m>0, \sigma \in \operatorname{Gal}(L / k) \backslash \operatorname{Gal}(L / \ell)$, and $v \in \operatorname{Aut}(E)$, we have $m P \in E(\ell)^{v^{-1} \sigma}$, or $\sigma(m P)=v m P$. From this it follows that $m P$ is a point on a twist of $E$, defined over $k^{\prime} \cap \ell$, where $k^{\prime}$ is the fixed field of $\sigma$.

At the time of writing, Conjecture 3.1 is known only in the following special case. See [McKinnon 2003] for a proof, and [Silverman 2005] for a discussion of the implications of Vojta's conjecture in this context.

Theorem 3.6 [McKinnon 2003]. Let $E$ be an elliptic curve over a number field $\ell$. Let $R=\operatorname{End}_{\ell}(E)$. Let $M$ be a cyclic R-submodule of $E(\ell)$. Then Conjecture 3.1 holds for $(P, Q) \in M \times M \subset(E \times E)(\ell)$; that is, in the notation of Conjecture 3.1, there exists a proper Zariski closed subset $Z(\epsilon)$ of $X$ such that for every $(P, Q) \in$ $M \times M-\pi(Z(\epsilon))$, we have

$$
h_{Y}\left(\pi^{-1}(P, Q)\right) \leq \epsilon(h(P)+h(Q))+O(1),
$$

where $h_{Y}$ is a logarithmic height function associated to the exceptional divisor on the blowup $X$ of $E \times E$ at a finite set of points and $h$ is any fixed ample logarithmic height on $E$. 
Theorem 3.7. Let $E$ be an elliptic curve over a number field $k$ with complex multiplication. Let $\ell$ be the compositum of $k$ with the imaginary quadratic field $\operatorname{End}_{\bar{k}}(E) \otimes \mathbb{Q}$. Let $D$ be a nontrivial effective divisor on $E$ defined over $\ell$. Let $P \in E(\ell)$ and $\epsilon>0$. Then either

$$
h_{D, \operatorname{deg}_{>1}(\ell / k)}(n P)<\epsilon h_{D}(n P)
$$

for all but finitely many $n>0$, or there exists a positive integer $m$, an elliptic curve $E^{\prime} / k$, and an isomorphism $\phi: E \rightarrow E^{\prime}$ over $\ell$ such that $\phi(m P)$ is a $k$-rational point on $E^{\prime}$.

Proof. If $\ell=k$ then the theorem is vacuous. So suppose that $\ell$ is a quadratic extension of $k$. Let $R=\operatorname{End}_{\bar{k}}(E)$. First, we note that $R[E(k)]$ has finite index in $E(\ell)$. Indeed, as is well-known [Silverman 1992, Exercise X-10.16], we have rk $E(\ell)=\mathrm{rk} E(k)+\mathrm{rk} E^{\prime}(k)$, where $E^{\prime}$ is a quadratic twist of $E$ over $\ell$. If $\ell=k(\sqrt{N})$, $N \in \mathbb{Z}$, then any element $n \sqrt{N} \in R$, with $n$ a positive integer, induces an isogeny (over $k$ ) between $E$ and a quadratic twist $E^{\prime}$ of $E$ over $\ell$. Thus, rk $E(k)=$ rk $E^{\prime}(k)$ and we have $\operatorname{rk} E(\ell)=2 \mathrm{rk} E(k)=\operatorname{rk} R[E(k)]$.

Next, we claim that Theorem 3.6 actually holds under the slightly weaker assumption that $M$ contains a cyclic $R$-submodule $M^{\prime}$ of finite index $m$. Indeed, one easily reduces to considering the case where $X$ is the blow-up of $E \times E$ at the origin $(\mathbb{O}, \mathcal{O})$ and $Y$ is the exceptional divisor. The claim then follows by applying Theorem 3.6 to $M^{\prime}$ and from the facts

$$
h_{Y}\left(\pi^{-1}(P, Q)\right) \leq h_{Y}\left(\pi^{-1}(m P, m Q)\right)+O(1),
$$

$(P, Q) \neq(\mathbb{O}, \mathcal{O})$, and $h(m P)=m^{2} h(P)+O(1)$.

Let $m$ be the index of $R[E(k)]$ in $E(\ell)$. Let $P \in E(\ell)$. Then we have $m P=\phi(Q)$, for some $Q \in E(k)$ and some $\phi \in R$. Let $\sigma$ be the unique nonidentity element of $\operatorname{Gal}(\ell / k)$. Then $m \sigma(P)=\sigma(m P)=\sigma(\phi(Q))=(\sigma \phi)(Q)$, so $m P$ and $m \sigma(P)$ both belong to the cyclic $R$-submodule $R Q$ of $E(\ell)$ generated by $Q$. So $R Q$ has finite index in the subgroup of $E(\ell)$ generated by $R Q, P$, and $\sigma(P)$. Then by our earlier claim, Conjecture 3.1 holds for the points $(n P, n \sigma(P)) \in(E \times E)(\ell), n \in \mathbb{Z}$. But now the same proof as in Theorem 3.4 and Corollary 3.5 works, completing the proof.

\section{References}

[Corvaja and Zannier 2005] P. Corvaja and U. Zannier, "A lower bound for the height of a rational function at S-unit points", Monatsh. Math. 144:3 (2005), 203-224. MR 2005k:11140 Zbl 1086. 11035

[McKinnon 2003] D. McKinnon, "Vojta's main conjecture for blowup surfaces", Proc. Amer. Math. Soc. 131:1 (2003), 1-12. MR 2003g:11071 Zbl 1022.11027 
[Silverman 1992] J. H. Silverman, The arithmetic of elliptic curves, Graduate Texts in Mathematics 106, Springer, New York, 1992. MR 95m:11054

[Silverman 2005] J. H. Silverman, "Generalized greatest common divisors, divisibility sequences, and Vojta's conjecture for blowups”, Monatsh. Math. 145:4 (2005), 333-350. MR 2006e:11087 Zbl 1197.11070

[Vojta 1987] P. Vojta, Diophantine approximations and value distribution theory, Lecture Notes in Mathematics 1239, Springer, Berlin, 1987. MR 91k:11049 Zbl 0609.14011

[Vojta 2011] P. Vojta, "Diophantine approximation and Nevanlinna theory", pp. 111-224 in Arithmetic geometry (Cetraro, 2007), edited by P. Corvaja and C. Gasbarri, Lecture Notes in Mathematics 2009, Springer, Berlin, 2011. MR 2012i:11076 Zbl 05882118

Communicated by Bjorn Poonen

Received 2011-06-02 Revised 2011-10-18 Accepted 2011-12-10

adlevin@math.msu.edu

Department of Mathematics, Michigan State University, East Lansing, MI 48824, United States

dmckinnon@math.uwaterloo.ca Department of Pure Mathematics, University of Waterloo, Waterloo, ON, N2T 2M2, Canada 


\section{Algebra \& Number Theory}

msp.berkeley.edu/ant

\section{EDITORS}

MANAGING EDITOR

Bjorn Poonen

Massachusetts Institute of Technology

Cambridge, USA

\author{
EDITORIAL BOARD CHAIR \\ David Eisenbud \\ University of California \\ Berkeley, USA
}

\section{BOARD OF EDITORS}

Georgia Benkart

Dave Benson

Richard E. Borcherds

John H. Coates

J-L. Colliot-Thélène

Brian D. Conrad

Hélène Esnault

Hubert Flenner

Edward Frenkel

Andrew Granville

Joseph Gubeladze

Ehud Hrushovski

Craig Huneke

Mikhail Kapranov

Yujiro Kawamata

János Kollár

Yuri Manin

Barry Mazur

Philippe Michel
University of Wisconsin, Madison, USA

University of Aberdeen, Scotland

University of California, Berkeley, USA

University of Cambridge, UK

CNRS, Université Paris-Sud, France

University of Michigan, USA

Universität Duisburg-Essen, Germany

Ruhr-Universität, Germany

University of California, Berkeley, USA

Université de Montréal, Canada

San Francisco State University, USA

Hebrew University, Israel

University of Kansas, USA

Yale University, USA

University of Tokyo, Japan

Princeton University, USA

Northwestern University, USA

Harvard University, USA

École Polytechnique Fédérale de Lausanne
Susan Montgomery

Shigefumi Mori

Raman Parimala

Jonathan Pila

Victor Reiner

Karl Rubin

Peter Sarnak

Joseph H. Silverman

Michael Singer

Vasudevan Srinivas

J. Toby Stafford

Bernd Sturmfels

Richard Taylor

Ravi Vakil

Michel van den Bergh

Marie-France Vignéras

Kei-Ichi Watanabe

Andrei Zelevinsky

Efim Zelmanov
University of Southern California, USA

RIMS, Kyoto University, Japan

Emory University, USA

University of Oxford, UK

University of Minnesota, USA

University of California, Irvine, USA

Princeton University, USA

Brown University, USA

North Carolina State University, USA

Tata Inst. of Fund. Research, India

University of Michigan, USA

University of California, Berkeley, USA

Harvard University, USA

Stanford University, USA

Hasselt University, Belgium

Université Paris VII, France

Nihon University, Japan

Northeastern University, USA

University of California, San Diego, USA

\section{PRODUCTION}

contact@msp.org

Silvio Levy, Scientific Editor

See inside back cover or www.jant.org for submission instructions.

The subscription price for 2012 is US \$175/year for the electronic version, and \$275/year (+ \$40 shipping outside the US) for print and electronic. Subscriptions, requests for back issues from the last three years and changes of subscribers address should be sent to Mathematical Sciences Publishers, Department of Mathematics, University of California, Berkeley, CA 94720-3840, USA.

Algebra \& Number Theory (ISSN 1937-0652) at Mathematical Sciences Publishers, Department of Mathematics, University of California, Berkeley, CA 94720-3840 is published continuously online. Periodical rate postage paid at Berkeley, CA 94704, and additional mailing offices.

ANT peer review and production are managed by EditFLOW ${ }^{\circledR}$ from Mathematical Sciences Publishers.

PUBLISHED BY

mathematical sciences publishers

http://msp.org/

A NON-PROFIT CORPORATION

Typeset in IATEX

Copyright $@ 2012$ by Mathematical Sciences Publishers 


\section{Algebra \& Number Theory}

Volume $6 \quad$ No. $6 \quad 2012$

The smallest prime that does not split completely in a number field

XIANNAN LI

On the geometric realization of the inner product and canonical basis for quantum affine

1097 $\mathfrak{s l}_{n}$

\section{KEVIN MCGERTY}

Combinatorics of the tropical Torelli map

MELODY CHAN

Sonia Natale and Julia Yael PlavniK

Cusp form motives and admissible $G$-covers

DAN PETERSEN

Ideals of degree one contribute most of the height

AARON LEVIN and DAVID MCKINNON

Torsion des modules de Drinfeld de rang 2 et formes modulaires de Drinfeld 